\title{
Modelo de Kirkwood-Fröhlich para fluidos polares puros
}

\section{Kirkwood-Fröhlich model for pure polar fluids}

\author{
Paulo Peixoto*1@, Adonias Barros ${ }^{2 @}$, Mario Henrique Oliveira ${ }^{3 ®}$ \\ ${ }^{1}$ Universidade Federal de Pernambuco, Núcleo de Formação Docente, Caruaru, PE, Brasil. \\ ${ }^{2}$ Universidade Federal de Pernambuco, Departamento de Física, Recife, PE, Brasil. \\ ${ }^{3}$ Universidade Federal Rural de Pernambuco, Unidade Acadêmica de Serra Talhada, Serra Talhada, PE, Brasil.
}

Recebido em 15 de julho de 2021. Aceito em 20 de setembro de 2021.

\begin{abstract}
Neste artigo, guiamos o leitor ou a leitora à obtenção da equação de Kirkwood-Fröhlich para fluidos polares puros. Tal equação relaciona a constante dielétrica do fluido, seu índice de refração, sua densidade, sua temperatura e o momento de dipolo permanente de suas moléculas constituintes, e envolve ainda um fator adimensional $g-$ conhecido como fator de correlação de Kirkwood - cujo valor é uma expressão da correlação entre as orientações das moléculas do fluido, na situação de campo elétrico externo nulo.

Palavras-chave: Constante dielétrica, dielétricos, modelo de Kirkwood-Fröhlich.
\end{abstract}

In this paper, we guide the reader to obtain the Kirkwood-Fröhlich equation for pure polar fluids. Such equation relates the dielectric constant of the fluid, its refractive index, its density, its temperature, and the permanent dipole moment of its constituent molecules, and also involves a dimensionless factor $g$ - known as Kirkwood correlation factor - whose value is an expression of the correlation between the orientantions of the molecules of the fluid, in the case of zero external electric field.

Keywords: Dielectric constant, dielectrics, Kirkwood-Fröhlich model.

\section{Introdução}

Este artigo é o terceiro de uma série sobre modelos para fluidos polares puros, e a conclui. No primeiro artigo [1], guiamos o leitor ou a leitora à obtenção da equação de Onsager [2] 1

$$
\frac{\left(\epsilon_{r}-n^{2}\right)\left(2 \epsilon_{r}+n^{2}\right)}{\epsilon_{r}\left(n^{2}+2\right)^{2}}=\frac{N}{3 \epsilon_{0}} \frac{p_{0}^{2}}{3 k T},
$$

que relaciona a constante dielétrica $\epsilon_{r}$ do fluido, seu índice de refração $n$, sua densidade numérica $N$, sua temperatura $T$ e o módulo $p_{0}$ do momento de dipolo permanente de suas moléculas constituintes. $k$ é a constante de Boltzmann, e $\epsilon_{0}$ a permissividade elétrica do vácuo. No percurso, mostramos que a equação de Onsager pode ser escrita como

$$
\begin{aligned}
\frac{\epsilon_{r}-1}{\epsilon_{r}+2}= & \frac{N \alpha}{3 \epsilon_{0}} \\
& +\left(\frac{9 \epsilon_{r} /\left(\epsilon_{r}+2\right)}{2 \epsilon_{r}+1-2\left(\epsilon_{r}-1\right) N \alpha / 3 \epsilon_{0}}\right) \frac{N}{3 \epsilon_{0}} \frac{p_{0}^{2}}{3 k T}
\end{aligned}
$$

quando escolhemos trabalhar com a polarizabilidade molecular $\alpha$, em vez de com o índice de refração $n$.

No mesmo artigo, discutimos o modelo de Debye [3] como resultado de uma aproximação de campo médio

\footnotetext{
* Endereço de correspondência: paulo.peixoto@ufpe.br

1 Mantém-se aqui nossa opção de escrevermos todas as equações no Sistema Internacional de Unidades (SI).
}

aplicada ao modelo de Onsager. Em uma parte da seção correspondente, mostramos que a equação de Debye, tradicionalmente apresentada (no SI) na forma

$$
\frac{\epsilon_{r}-1}{\epsilon_{r}+2}=\frac{N}{3 \epsilon_{0}}\left(\alpha+\frac{p_{0}^{2}}{3 k T}\right)
$$

pode ser reescrita - trabalhando-se com $n$, em vez de $\alpha-$ como

$$
\frac{3\left(\epsilon_{r}-n^{2}\right)}{\left(\epsilon_{r}+2\right)\left(n^{2}+2\right)}=\frac{N}{3 \epsilon_{0}} \frac{p_{0}^{2}}{3 k T} .
$$

Perceba que são mais adequadas a comparações as equações (1) e (4), e as equações (2) e (3), pois cada um desses dois pares de equações envolve as mesmas grandezas físicas. Fica evidente, a partir de (2) e (3), que as equações de Onsager e Debye recaem na equação de Clausius-Mossotti [4,

$$
\frac{\epsilon_{r}-1}{\epsilon_{r}+2}=\frac{N \alpha}{3 \epsilon_{0}}
$$

no caso particular de moléculas apolares $\left(p_{0}=0\right)$.

Os modelos de Onsager e Debye podem ser classificados como modelos com aproximação de meio contínuo, porque neles uma única molécula do fluido é modelada como um dipolo em uma cavidade esférica microscópica, e além daquela cavidade o dielétrico é tratado como um meio contínuo (veja a Figura 1). Trata-se de uma aproximação um tanto grosseira, como discutimos no 


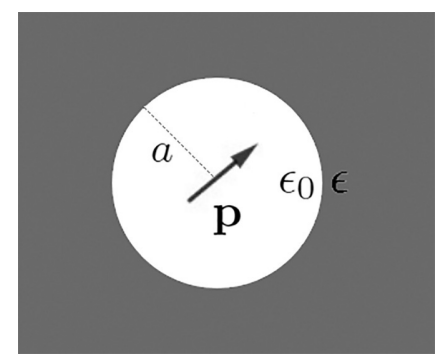

Figura 1: Cavidade esférica microscópica imaginária, de raio $a$, em um fluido dielétrico (nos modelos com aproximação de meio contínuo). A cavidade contém uma única molécula do fluido, aqui representada por seu momento de dipolo total $\mathbf{p}$ (em um determinado instante). Além da cavidade, o dielétrico é modelado como um meio contínuo de permissividade $\epsilon$.

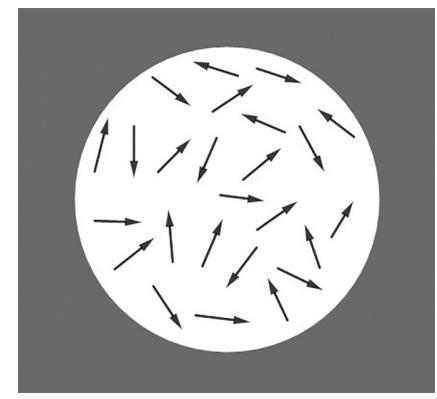

Figura 2: Esfera macroscópica imaginária relativa ao modelo de Kirkwood para fluidos polares puros. As $\mathcal{N}$ moléculas dentro da esfera são tratadas como dipolos permanentes (explicitados, aqui, em número bastante reduzido, é claro), e fora dela o dielétrico é modelado como um meio contínuo.

primeiro artigo [1], mas que, sendo parte desses dois modelos, nos ensina muito sobre modelagem em física.

Como dissemos em nosso segundo artigo desta série [5], Kirkwood buscou melhorar a modelagem feita por Onsager substituindo a cavidade microscópica da Fig. 11 por uma esfera macroscópica, ilustrada na Fig. 2 . No modelo de Kirkwood [6], as moléculas do fluido dielétrico são tratadas de forma discreta no interior dessa esfera macroscópica, de raio $a_{\text {macro, }}$ e apenas além dela o fluido é modelado como um meio contínuo. Dissemos também que, por outro lado, uma limitação do modelo de Kirkwood (posteriormente contornada, de forma bastante criativa, por Fröhlich [7, como veremos na próxima seção) é que seu desenvolvimento se aplica apenas a moléculas não polarizáveis (ou com polarizabilidades desprezíveis), e, assim, cada molécula no interior da esfera macroscópica da Fig. 22 é descrita apenas como um momento de dipolo permanente, de módulo $p_{0}$.

Mas por que o desenvolvimento que culmina na equação de Kirkwood (todos os detalhes foram apresentados em nosso segundo artigo [5]),

$$
\frac{\left(\epsilon_{r}-1\right)\left(2 \epsilon_{r}+1\right)}{9 \epsilon_{r}}=\frac{N}{3 \epsilon_{0}} \frac{p_{0}^{2}}{3 k T} g,
$$

sendo $g$ o chamado fator de correlação de Kirkwood (ou fator $g$ de Kirkwood), definido como

$$
g \equiv \frac{1}{\mathcal{N}} \sum_{i=1}^{\mathcal{N}} \sum_{j=1}^{\mathcal{N}} \overline{\left(\cos \theta_{i j}\right)_{0}},
$$

em que $\overline{\left(\cos \theta_{i j}\right)_{0}}$ é a média do cosseno do ângulo entre dois dipolos $i$ e $j$ na esfera macroscópica da Fig. 2, na situação de campo externo nulo (indicada pelo subscrito "0") ${ }^{2}$ não se aplica a moléculas com polarizabilidades não desprezíveis? Simples: o módulo do momento de dipolo de cada molécula do fluido não seria mais constante (devido ao momento de dipolo induzido), e assim não estaria correto um desenvolvimento como este, apresentado em nosso segundo artigo [5] (sendo $\mathbf{M}$ o momento de dipolo total da porção do fluido na esfera ilustrada na Fig. 2):

$$
\begin{aligned}
\overline{\left(M^{2}\right)_{0}} & =\overline{(\mathbf{M} \cdot \mathbf{M})_{0}}=\overline{\left[\left(\sum_{i=1}^{\mathcal{N}} \mathbf{p}_{\mathbf{0}_{\mathbf{i}}}\right) \cdot\left(\sum_{j=1}^{\mathcal{N}} \mathbf{p}_{\mathbf{0}_{\mathbf{j}}}\right)\right]_{0}} \\
& =\overline{(\sum_{i=1}^{\mathcal{N}} \sum_{j=1}^{\mathcal{N}} \overbrace{\mathbf{p}_{0}^{2} \cdot \cos \theta_{i j}}^{\mathbf{p}_{\mathbf{0}_{\mathbf{j}}}})}=p_{0}^{2} \sum_{i=1}^{\mathcal{N}} \sum_{j=1}^{\mathcal{N}} \overline{\left(\cos \theta_{i j}\right)_{0}} \\
& =\mathcal{N} p_{0}^{2} g .
\end{aligned}
$$

Como foi, então, que Fröhlich contornou essa dificuldade? Veremos na próxima seção.

\section{Obtendo a Equação de Kirkwood-Fröhlich}

Para contornar a dificuldade de incluir momentos de dipolo induzidos no desenvolvimento que leva à equação de Kirkwood [5], Fröhlich [7 modelou uma molécula do fluido, posta no vácuo, separada das demais, como um dipolo puro, com momento de dipolo constante $\mathbf{p}_{\mathbf{d}}$, no centro de uma esfera homogênea constituída de um meio contínuo apolar com permissividade elétrica $\epsilon_{\text {esf }}$ e diâmetro da ordem da distância média entre duas moléculas adjacentes no fluido (explicaremos isso), como ilustra a Fig. 3 Mais adiante, nesta seção, mostraremos por que essa foi uma ideia extremamente criativa, mas, antes, vamos discutir esse modelo de Fröhlich para uma molécula do fluido, posta no vácuo, separada das demais.

Em primeiro lugar, no caso em que o fluido é um líquido típico, a distância média entre duas moléculas adjacentes é da ordem do diâmetro molecular. Isso se aplica à água, por exemplo. Neste caso, portanto, o diâmetro da esfera ilustrada na Fig. 3 é da ordem do diâmetro da molécula que ela representa. Como é possível, então, que tal esfera seja constituída de um

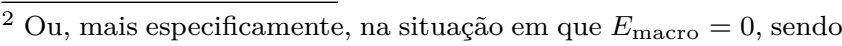
$\mathbf{E}_{\text {macro }}$ o campo que age sobre as moléculas na esfera macroscópica da Fig. 2
} 


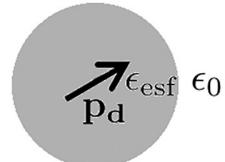

(a)

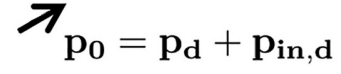

(b)
Figura 3: (a) Modelo de uma molécula no vácuo, criado por Fröhlich especificamente para a inclusão de polarizabilidades não nulas no modelo de Kirkwood, constituído de um dipolo puro, com momento de dipolo constante $\mathbf{p}_{\mathbf{d}}$, no centro de uma esfera homogênea constituída de um meio contínuo apolar com permissividade $\epsilon_{\text {esf }}$ e diâmetro da ordem da distância média entre duas moléculas adjacentes no fluido. (b) $\mathrm{O}$ momento de dipolo permanente da molécula, $\mathbf{p}_{\mathbf{0}}$, é igual $\mathbf{p}_{\mathbf{d}}+\mathbf{p}_{\mathbf{i n}, \mathbf{d}}$, em que $\mathbf{p}_{\mathbf{i n}, \mathbf{d}}$ é o momento de dipolo induzido, na esfera, pelo dipolo puro com momento de dipolo constante $\mathbf{p}_{\mathbf{d}}$.

meio contínuo apolar (lembrando que um meio contínuo apolar é um meio macroscópico constituído de moléculas apolares - ou seja, sem momento de dipolo permanente)? Claro que não é possível. Logo, Fröhlich aplicou a eletrodinâmica de meios contínuos (para o cálculo da relação, que obteremos mais adiante, entre $\mathbf{p}_{\mathbf{0}}$ e $\mathbf{p}_{\mathbf{d}}-$ veja a Fig. 3b) considerando dimensões às quais, claramente, ela não se aplica. E isso é parte de seu modelo, por mais estranho que pareça (e, de fato, é). E não foi algo muito diferente do que fez Onsager ao considerar, em seu modelo, uma cavidade esférica microscópica, ilustrada na Fig. 1] cercada por um meio contínuo - o que é um tanto irônico, já que o modelo de Kirkwood-Fröhlich tem, em parte, a pretensão de superar esse tipo de limitação do modelo de Onsager ${ }^{3}$

O momento de dipolo total de uma molécula do fluido, posta no vácuo, separada das demais, é seu momento de dipolo permanente $\mathbf{p}_{\mathbf{0}}$ (pois não há nenhum momento de dipolo induzido por um campo externo). Assim, no modelo de Fröhlich (Fig. 3), $\mathbf{p}_{\mathbf{0}}$ é igual à soma do momento de dipolo $\mathbf{p}_{\mathbf{d}}$ com o momento de dipolo $\mathbf{p}_{\mathbf{i n}, \mathbf{d}}$ induzido, na esfera, pelo dipolo puro com momento de dipolo constante $\mathbf{p}_{\mathbf{d}}$ :

$$
\mathbf{p}_{\mathbf{0}}=\mathbf{p}_{\mathbf{d}}+\mathbf{p}_{\mathrm{in}, \mathrm{d}}
$$

Vamos mostrar que a eletrodinâmica de meios contínuos nos dá a seguinte relação entre $\mathbf{p}_{\mathbf{d}}$ e $\mathbf{p}_{\mathbf{0}}$ :

$$
\mathbf{p}_{\mathbf{d}}=\frac{\left(\epsilon_{\mathrm{esf}}\right)_{r}+2}{3} \mathbf{p}_{\mathbf{0}}
$$

em que $\left(\epsilon_{\text {esf }}\right)_{r} \equiv \epsilon_{\text {esf }} / \epsilon_{0}$ é a constante dielétrica (ou permissividade relativa) do meio de permissividade $\epsilon_{\text {esf }}$. Antes de partirmos para as contas, deve ficar claro que a igualdade (9) nos dá o momento de dipolo constante $\mathbf{p}_{\mathbf{d}}$ que gera, na esfera homogênea da Fig. 3, um momento

\footnotetext{
3 Se você é um estudante ou uma estudante de física, esperamos que, a essa altura, já tenha abandonado - se é que já teve - a ideia de que a física é uma ciência exata.
}

de dipolo induzido $\mathbf{p}_{\mathbf{i n}, \mathbf{d}}$ tal que a soma $\mathbf{p}_{\mathbf{d}}+\mathbf{p}_{\mathbf{i n}, \mathbf{d}}$ é igual ao momento de dipolo total da molécula isolada, no vácuo - que é seu momento de dipolo permanente $\mathbf{p}_{0} \unlhd^{4}$

Escolhendo, como o eixo $z$, o eixo com a mesma direção e o mesmo sentido de $\mathbf{p}_{\mathbf{d}}$, na Fig. 3 temos um problema de cálculo de potencial elétrico com simetria azimutal, cuja solução geral é:

$$
V(r, \theta, \phi)=V(r, \theta)=\sum_{l=0}^{\infty}\left(A_{l} r^{l}+\frac{B_{l}}{r^{l+1}}\right) P_{l}(\cos \theta),
$$

em que as funções $P_{l}$ são os polinômios de Legendre $\left(P_{0}(x)=1, P_{1}(x)=x, P_{2}(x)=\left(3 x^{2}-1\right) / 2, \ldots\right)$. Sendo $a$ o raio da esfera da Fig. 3 temos, então,

$$
V_{\mathrm{esf}}(r, \theta)=\sum_{l=0}^{\infty}\left(A_{l} r^{l}+\frac{B_{l}}{r^{l+1}}\right) P_{l}(\cos \theta) \quad(\text { para } r \leq a),
$$

e, fora da esfera,

$V_{\text {fora }}(r, \theta)=\sum_{l=0}^{\infty}\left(C_{l} r^{l}+\frac{D_{l}}{r^{l+1}}\right) P_{l}(\cos \theta) \quad($ para $r \geq a)$.

São quatro as condições de contorno para este problema. Uma delas,

$$
V_{\text {esf }}(a, \theta)=V_{\text {fora }}(a, \theta),
$$

é a continuidade do potencial elétrico ao passarmos da esfera para o meio contínuo $5^{5}$ Outra condição de contorno que também se aplica à transição da cavidade para o meio contínuo é

$$
\left.\epsilon_{\mathrm{esf}} \frac{\partial V_{\mathrm{esf}}}{\partial r}\right|_{r=a}=\left.\epsilon_{0} \frac{\partial V_{\mathrm{fora}}}{\partial r}\right|_{r=a},
$$

que vem da condição mais geral $\epsilon_{\text {abaixo }}\left(\partial V_{\text {abaixo }} / \partial n\right)-$ $\epsilon_{\text {acima }}\left(\partial V_{\text {acima }} / \partial n\right)=\sigma_{f}$, em que $n$ denota uma coordenada normal à superfície de transição de um meio para o outro e $\sigma_{f}$ é a densidade superficial de carga livre nessa superfície (veja, por exemplo, a seção 4.4.2 (Boundary Value Problems with Linear Dielectrics) do livro do Griffiths 8]). A próxima condição de contorno diz respeito à aproximação esperada para $V_{\text {esf }}$ quando temos $r \ll a$ :

$$
V_{\mathrm{esf}}(r, \theta) \approx \frac{1}{4 \pi \epsilon_{\mathrm{esf}}} \frac{p_{d} \cos \theta}{r^{2}}, \text { para } r \ll a .
$$

Trata-se da expressão para o potencial de um dipolo puro, com momento de dipolo de módulo $p_{d}$, mergulhado em um meio linear contínuo de permissividade $\epsilon_{\text {esf }}$.

\footnotetext{
${ }^{4}$ A partir das igualdades 8 e 90 obtemos $\mathbf{p}_{\mathbf{i n}, \mathbf{d}}=\frac{1-\left(\epsilon_{\text {esf }}\right)_{r}}{3} \mathbf{p}_{\mathbf{0}}$ Perceba que os momentos de dipolo $\mathbf{p}_{\mathbf{i n}, \mathbf{d}}$ e po têm a mesma direção, mas sentidos opostos. Isto explica por que a seta que representa o vetor $\mathbf{p}_{\mathbf{0}}$, na Fig. 3 é menor que a seta que representa, na mesma figura, o vetor $\mathbf{p}_{\mathbf{d}}$

${ }^{5}$ Note que essa condição de contorno foi implicitamente assumida acima quando afirmamos que as expressões para $V_{\text {esf }}(r, \theta)$ e para $V_{\text {fora }}(r, \theta)$ valem, ambas, para $r=a$.
} 
A última condição de contorno diz respeito à aproximação esperada para $V_{\text {fora }}$ quando temos $r \gg a$ :

$$
V_{\text {fora }}(r, \theta) \approx \frac{1}{4 \pi \epsilon_{0}} \frac{p_{0} \cos \theta}{r^{2}}, \text { para } r \gg a .
$$

Você entende bem esta última condição de contorno? Muito distante da esfera da Fig. 3, o potencial gerado pela esfera e pelo dipolo puro com momento de dipolo $\mathbf{p}_{\mathbf{d}}$ é aproximadamente igual ao potencial de um dipolo puro, com momento de dipolo igual ao momento de dipolo total do sistema - que é o momento de dipolo permanente da molécula, cujo módulo é $p_{0}$. Mas $p_{0}$ é uma constante a ser relacionada a $p_{d}$ e $\epsilon_{\text {esf }}$. É tal relação que nos interessa, aqui.

Pois bem, aplicando essas quatro condições de contorno às expressões gerais acima para $V_{\text {esf }}(r, \theta)$ e/ou $V_{\text {fora }}(r, \theta)$, obtemos 6

$$
p_{0}=\frac{3 p_{d}}{\left(\epsilon_{\mathrm{esf}}\right)_{r}+2},
$$

de onde segue a igualdade (9).

Então, recapitulando, temos na Fig. 3 o modelo de Fröhlich para uma molécula do fluido posta no vácuo, separada das demais. Tal modelo consiste em um dipolo puro, com momento de dipolo constante $\mathbf{p}_{\mathbf{d}}$ dado pela igualdade (9) (que garante que o momento de dipolo total do sistema é igual ao momento de dipolo permanente $\mathbf{p}_{\mathbf{0}}$ da molécula), no centro de uma esfera constituída de um meio contínuo apolar, de permissividade $\epsilon_{\text {esf }}$, sendo o diâmetro dessa esfera da ordem da distância média entre duas moléculas adjacentes no fluido.

Vamos explicar a seguir por que no modelo apresentado na Fig. 3 o meio homogêneo que constitui a esfera é apolar e por que seu diâmetro é da ordem da distância média entre moléculas adjacentes no fluido (com a distância entre duas moléculas medida a partir de seus centros de massa).

Sendo apolar o meio de permissividade $\epsilon_{\text {esf }}$, é válida a relação

$$
\left(\epsilon_{\mathrm{esf}}\right)_{r}=n^{2}
$$

em que $n$ é o índice de refração daquele meio e $\left(\epsilon_{\mathrm{esf}}\right)_{r}$ é sua constante dielétrica estática. Vejamos com mais detalhes. O índice de refração $n$ de um meio é definido como a razão entre a velocidade da luz no vácuo $(c)$ e a velocidade $v$ da luz nesse meio; ou seja, $n \equiv c / v$. De acordo com a eletrodinâmica clássica, $c=1 / \sqrt{\mu_{0} \epsilon_{0}}$, e, para meios lineares, $v=1 / \sqrt{\mu \epsilon}$ (veja, por exemplo, as seções 9.2.1 e 9.3.1 do Griffiths [8]). Como, para a maioria dos materiais, a permeabilidade magnética, $\mu$, é muito próxima da permeabilidade magnética do vácuo, $\mu_{0}$ (veja, por exemplo, a Tabela 6.1 do Griffiths [8]),

6 O nível de dificuldade aqui corresponde ao de um problema típico de uma lista de exercícios de uma disciplina de graduação de eletrodinâmica clássica. temos, para esses materiais, $n \approx \sqrt{\epsilon / \epsilon_{0}}=\sqrt{\epsilon_{r}}$, ou, equivalentemente, $\epsilon_{r}=n^{2}$ (escrevendo "=" no lugar de " $\approx "$ "). Mas atenção: a constante dielétrica $\epsilon_{r}$ é uma constante no sentido de que seu valor não depende da intensidade do campo elétrico macroscópico $\mathbf{E}$ (desde que, é claro, tal intensidade seja suficientemente baixa para que o meio possa ser classificado como linear). Contudo, seu valor pode depender da frequência do campo elétrico $\mathbf{E}$ ! Na relação $\epsilon_{r}=n^{2}$, temos $\epsilon_{r}=\epsilon_{r}(\nu)$, em que $\nu$ é a frequência de $\mathbf{E}$ (tipicamente uma frequência óptica), enquanto nas equações de Debye, Onsager, Kirkwood e Kirkwood-Fröhlich temos $\epsilon_{r}=\epsilon_{r}(0)-$ e neste caso a constante dielétrica é denominada constante dielétrica estática. Quando um meio dielétrico linear é submetido a um campo elétrico que oscila com frequência óptica, os momentos de dipolo permanentes não acompanham as oscilações do campo, e, assim, é como se eles não existissem! Isso porque as orientações moleculares ficam com uma distribuição aleatória, e então os momentos de dipolo permanentes não contribuem para a polarização do meio. Nesses casos, só os momentos de dipolo induzidos contribuem para o valor da constante dielétrica e por isso o valor de $\epsilon_{r}$ em frequências ópticas pode ser tão menor que seu valor para campos estáticos (no caso da água pura a $20^{\circ} \mathrm{C}$, submetida a luz visível, temos $\epsilon_{r} \approx 1,77$, e para campos elétricos estáticos temos $\epsilon_{r} \approx$ 80). Mas se o meio é um fluido linear e apolar (ou seja que não possui momentos de dipolo permanentes), sua constante dielétrica, com boa aproximação, independe da frequência - na faixa que vai de 0 a frequências ópticas -, e daí podemos usar a relação em 10 sendo $n$ o índice de refração do meio para frequências ópticas, mas sendo $\left(\epsilon_{\text {esf }}\right)_{r}$ sua constante dielétrica estática. Tal relação será fundamental na obtenção da equação de Kirkwood-Fröhlich.

Nosso próximo passo será reunir moléculas de um mesmo tipo, usando o modelo de Fröhlich apresentado na Fig. 3, para formar um fluido. A Fig 4 a ilustra a ideia, para um nível máximo de aproximação entre as moléculas. É claro, teríamos, com esse nível máximo de aproximação, uma estrutura regular característica de um sólido, não de um líquido. Mas o intento, aqui, é duplo: justificar por que o diâmetro da esfera, no modelo apresentado na Fig. 3 é da ordem da distância média entre moléculas adjacentes no fluido (com a distância entre duas moléculas medida a partir de seus centros de massa), e mostrar que mesmo nessa situação limite o espaço não é completamente preenchido - o que não é nenhuma surpresa, nem um problema, em princípio. Contudo, Fröhlich introduziu uma aproximação adicional: preencheu tais lacunas com o meio apolar de permissividade $\epsilon_{\text {esf }}$, como ilustra a Fig $4 \mathrm{~b}, 7 \mathrm{E}$ por que ele fez isso? Bem, é aqui que a mágica acontece (no melhor

\footnotetext{
7 É interessante observar que Onsager, no desenvolvimento de seu modelo, fez uso de uma aproximação semelhante: considerou o volume do fluido igual a uma soma de volumes esféricos. Veja a nota de rodapé 13 do primeiro artigo desta série [1].
} 
(a)

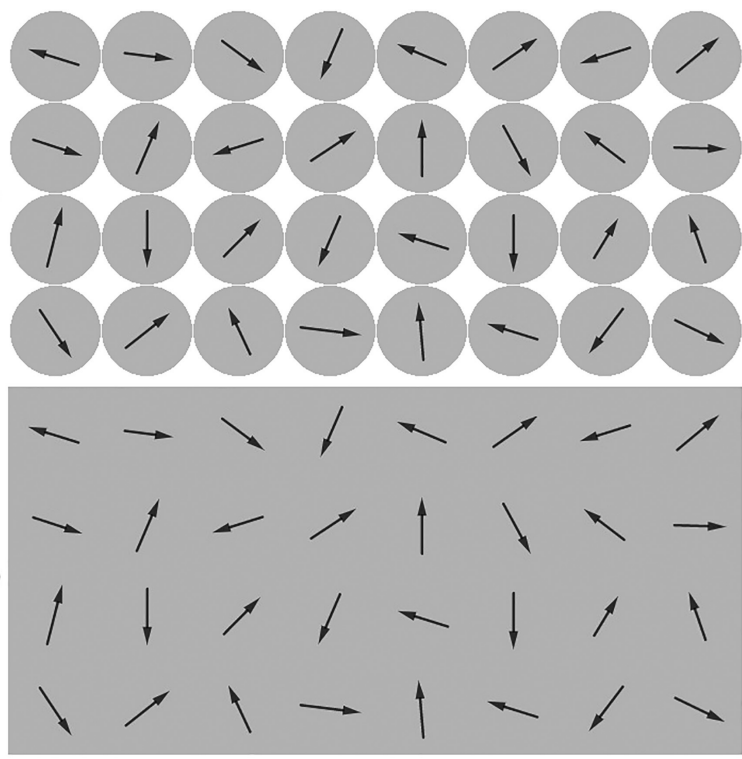

Figura 4: (a) Representação de um fluido puro constituído de moléculas segundo o modelo de Fröhlich (Fig. 3). Nesta ilustração, foi considerado o nível máximo de aproximação entre as moléculas - que não ocorre em líquidos. (b) A mesma distribuição de dipolos puros, com momentos de dipolo de módulo constante $p_{d}$, mas agora considerando-se que o meio de permissividade $\epsilon_{\text {esf }}$ preenche os espaços vazios.

sentido da expressão)! A grande ideia de Fröhlich, para contornar a dificuldade (discutida na seção anterior) de introduzir polarizabilidades moleculares não desprezíveis no desenvolvimento que leva à equação de Kirkwood, foi considerar, na esfera macroscópica ilustrada na Fig. 2, moléculas modeladas segundo ilustra a Fig. 3 . com a aproximação adicional de preencher os espaços vazios com o meio de permissividade $\epsilon_{\text {esf }}$. Com isso e exatamente aqui está a grande ideia de Fröhlich temos um conjunto de dipolos puros, com momentos de dipolo de módulo constante $p_{d}$, em um meio contínuo de permissividade $\epsilon_{\text {esf }}$. É o que ilustra a Fig. 5 Daí, a dificuldade relativa à existência de momentos de dipolo induzidos some, porque $p_{d}$ é constante, e o meio contínuo de permissividade $\epsilon_{\text {esf }}$ responde, no modelo, pelos momentos de dipolo induzidos. Então todo o desenvolvimento que leva à equação de Kirkwood, apresentado no segundo artigo desta série [5], pode ser mantido, fazendo-se apenas as seguintes modificações (compare as Figs.2 e 5): substituindo-se $\epsilon_{0}$ por $\epsilon_{\text {esf }}$ (como permite a eletrodinâmica, quando passamos do vácuo para um meio linear de permissividade $\epsilon_{\text {esf }}$ ) e $p_{0}$ por $p_{d}$. O resultado é que a equação de Kirkwood (igualdade (38) do segundo artigo desta série, e igualdade (6) deste artigo), torna-se:

$$
\frac{\left(\left(\epsilon / \epsilon_{\text {esf }}\right)-1\right)\left(2\left(\epsilon / \epsilon_{\text {esf }}\right)+1\right)}{9\left(\epsilon / \epsilon_{\text {esf }}\right)}=\frac{N}{3 \epsilon_{0}} \frac{p_{d}^{2}}{3 k T} g .
$$

Não substituímos, no segundo membro da igualdade (6), $\epsilon_{0}$ por $\epsilon_{\text {esf }}$ porque aquele $\epsilon_{0}$ vem da relação geral

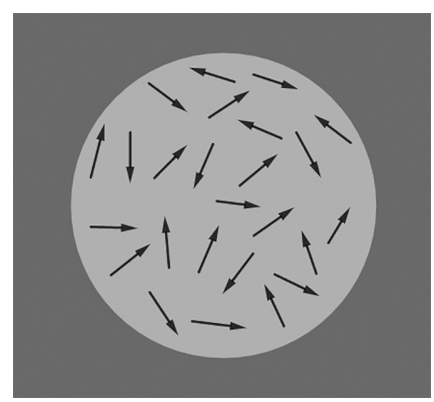

Figura 5: Esfera macroscópica imaginária relativa ao modelo de Kirkwood-Fröhlich para fluidos polares puros. O que muda, em relação à esfera apresentada na Fig. 2 é que aqui os dipolos permanentes têm momento de dipolo de módulo constante $p_{d}$, em vez de $p_{0}$ (veja a Fig. 3), e não estão no vácuo, mas imersos em um meio apolar contínuo de permissividade $\epsilon_{\text {esf }}$.

$\epsilon_{r}=1+P /\left(\epsilon_{0} E\right)$, como você pode facilmente verificar em nosso artigo anterior [5].

Combinando as igualdades (9), (10) e (11) (troque, em (11), $\epsilon / \epsilon_{\text {esf }}$ por $\left.\left(\epsilon / \epsilon_{0}\right) /\left(\epsilon_{\text {esf }} / \epsilon_{0}\right)=\epsilon_{r} /\left(\epsilon_{\text {esf }}\right)_{r}\right)$ obtemos a equação de Kirkwood-Fröhlich,

$$
\frac{\left(\epsilon_{r}-n^{2}\right)\left(2 \epsilon_{r}+n^{2}\right)}{\epsilon_{r}\left(n^{2}+2\right)^{2}}=\frac{N}{3 \epsilon_{0}} \frac{p_{0}^{2}}{3 k T} g .
$$

Embora $n$ tenha sido introduzido, no desenvolvimento apresentado acima, como o índice de refração do meio apolar de permissividade elétrica $\epsilon_{\text {esf }}$, é também o índice de refração do fluido dielétrico modelado, de permissividade $\epsilon$, porque os dipolos com momentos de dipolo de módulo constante $p_{d}$ não acompanham as oscilações, em frequências ópticas, de um campo elétrico aplicado ao meio, devido à inércia rotacional de cada molécula.

A equação de Kirkwood-Fröhlich, 12, difere da equação de Onsager, (1), pela presença, na primeira, do fator $g$, e a discussão feita no segundo artigo desta série [5], relativamente ao fator $g$ de Kirkwood, permanece válida aqui.

Vamos aplicar a equação de Kirkwood-Fröhlich à água. O momento de dipolo permanente de uma molécula de água tem módulo $p_{0} \approx 6,14 \times 10^{-30} \mathrm{C} \cdot \mathrm{m}$, e para água pura a $1 \mathrm{~atm}$ e $20^{\circ} \mathrm{C}(T=293 \mathrm{~K})$ temos $N \approx 3,34 \times 10^{28} \mathrm{~m}^{-3}$. Com estes valores, e com o valor calculado recentemente por Zhang e colaboradores [9] para o fator $g$ de Kirkwood para a água líquida, $g \approx 2,2$, a quantidade adimensional no segundo membro de 12 fica:

$$
\frac{N}{3 \epsilon_{0}} \frac{p_{0}^{2}}{3 k T} g \approx 8,60
$$

Substituindo o valor acima no segundo membro da igualdade $(12$, e fazendo, no primeiro membro, $n \approx 1,33$ (que é, aproximadamente, o índice de refração para a água líquida), resolvendo a equação resultante para $\epsilon_{r}$ obtemos $\epsilon_{r} \approx 62,0$. É um valor ainda distante do 
valor experimental para a constante dielétrica da água $\left(\epsilon_{r} \approx 80\right)$, mas é bem melhor que o valor que obtemos a partir da equação de Onsager (igualdade (1)), que é $\epsilon_{r} \approx 28,7$. É interessante observar que obtemos, a partir da equação de Kirkwood (igualdade (6p) - ou seja, sem considerar a polarizabilidade da molécula de água (que corresponde a fazer $n=1)-, \epsilon_{r} \approx 39,2$, e a partir da equação de Onsager com $n=1, \epsilon_{r} \approx 18,1$.

\section{Conclusão}

Neste trabalho, guiamos o leitor ou a leitora à obtenção da equação de Kirkwood-Fröhlich para fluidos polares puros - a equação (12). Como dissemos na primeira seção, trata-se do terceiro artigo de uma série sobre modelos para fluidos polares puros, e a conclui.

Esta série pode ser útil de formas variadas: para os que se interessam por teoria de polarização elétrica (para estudo ou realização de um trabalho de pesquisa), no ensino de tópicos complementares em disciplinas de eletrodinâmica clássica, mecânica estatística ou físicoquímica, em cursos de graduação ou pós-graduação, e também como um meio de discutir modelagem em física. As aproximações e considerações feitas no desenvolvimento dos modelos apresentados nesta série de artigos vão do curioso ao surpreendente.

\section{Agradecimentos}

Agradecemos à FACEPE pela bolsa de Iniciação Científica (IC) concedida a Adonias Barros. Na data de submissão deste artigo, Adonias já estava cursando mestrado em física (em outra área), mas este trabalho é, em parte, fruto do que foi produzido em seu período de IC.

\section{Referências}

[1] A. Barros e P. Peixoto, Revista Brasileira de Ensino de Física 42, e20200152 (2020).

[2] L. Onsager, Journal of the American Chemical Society 58, 1486 (1936).

[3] P. Debye, Physik. Zeits. 13, 97 (1912).

[4] C.J.F. Bottcher, Theory of Electric Polarization (Elsevier, Amsterdam, 1973), $2^{a}$ ed.

[5] P. Peixoto, A. Barros e M.H. Oliveira, Revista Brasileira de Ensino de Física 43, e20210219 (2021).

[6] J.G. Kirkwood, The Journal of Chemical Physics 7, 911 (1939).

[7] H. Fröhlich, Theory of Dielectrics - Dielectric Constant and Dielectric Loss (Clarendon Press, Oxford, 1949).

[8] D.J. Griffiths, Introduction to Electrodynamics (Cambridge University Press, Cambridge, 2017), $4^{a}$ ed.

[9] C. Zhang, J. Hutter e M. Sprik, The Journal of Physical Chemistry Letters 7, 2696 (2016). 